\title{
Preoperative opioid, sedative, and antidepressant use is associated with increased postoperative hospital costs in colorectal surgery
}

\author{
Nicholas A. Jackson ${ }^{1} \cdot$ Tong Gan $^{1}$ - Daniel L. Davenport ${ }^{2}$ - Doug R. Oyler²,3 Laura M. Ebbitt $^{4} \cdot$ B. Mark Evers ${ }^{2,3,5}$. \\ Avinash S. Bhakta ${ }^{2,3,6,7}$ (B)
}

Received: 28 July 2020 / Accepted: 29 September 2020 / Published online: 9 October 2020

(c) Springer Science+Business Media, LLC, part of Springer Nature 2020

\begin{abstract}
Background Opioid (OPD), sedative (SDT), and antidepressant (ADM) prescribing has increased dramatically over the last 20 years. This study evaluated preoperative OPD, SDT, and ADM use on hospital costs in patients undergoing colorectal resection at a single institution.

Methods This study was a retrospective record review. The local ACS-NSQIP database was queried for adult patients (age $\geq 18$ years) undergoing open/laparoscopic, partial/total colectomy, or proctectomy from January 1, 2013 to December 31, 2016. Individual patient medical records were reviewed to determine preoperative OPD, SDT, and AD use. Hospital cost data from index admission were captured by the hospital cost accounting system and matched to NSQIP query-identified cases. All ACS-NSQIP categorical patient characteristic, operative risk, and outcome variables were compared in medication groups using chi-square tests or Fisher's exact tests, and continuous variables were compared using Mann-Whitney U tests. Results A total of 1185 colorectal procedures were performed by 30 different surgeons. Of these, $27.6 \%$ patients took OPD, $18.5 \%$ SDT, and 27.8\% ADM preoperatively. Patients taking OPD, SDT, and ADM were found to have increased mean total hospital costs (MTHC) compared to non-users (30.8 vs 23.6 for OPD, 31.6 vs 24.4 for SDT, and 30.7 vs 23.8 for ADM). OPD and SDT use were identified as independent risk factors for increased MTHC on multivariable analysis.

Conclusion Preoperative OPD and SDT use can be used to predict increased MTHC in patients undergoing colorectal resections.
\end{abstract}

Keywords Opioids $\cdot$ Prescription drug use $\cdot$ Colorectal surgery $\cdot$ Healthcare costs $\cdot$ ACS-NSQIP

Avinash S. Bhakta

avinash.bhakta@uky.edu

1 Graduate Medical Education, General Surgery Residency Program, University of Kentucky, Lexington, KY, USA

2 Department of Surgery, University of Kentucky, Lexington, KY, USA

3 Division of General Surgery, University of Kentucky, Lexington, KY, USA

4 Department of Pharmacy Services, University of Kentucky, Lexington, KY, USA

5 Markey Cancer Center, University of Kentucky, Lexington, KY, USA

6 Section of Colorectal Surgery, University of Kentucky, Lexington, KY, USA

7 University of Kentucky Medical Center, 800 Rose St., C-233, Lexington, KY 40536, USA
Opioid, sedative, and antidepressant prescribing has increased dramatically over the last 20 years, and their use is now considered at the category of an epidemic. The use of opioids alone has seen an increase of $500 \%$ over that timeframe, and sedative medications have seen a nearly $50 \%$ increase, particularly in older patients [1]. Roughly one in eight Americans takes an antidepressant and antidepressant prescribing has seen an increase of over 65\% [2]. The Centers for Disease Control and Prevention now considers prescription drug use as one of the top five health challenges in the United States [3-5].

Healthcare costs have seen a similar increase. High-risk opioid users have increased overall healthcare costs by placing a nearly \$29 billion additional burden on healthcare [6-8]. Preoperative opioid use is prevalent in $25 \%$ of patients presenting for non-cardiac surgery and has recently been shown to increase hospital costs, as well as postoperative 
costs, in patients undergoing elective general surgery procedures $[9,10]$.

The cost of surgery and postoperative admission is taking on a larger focus. Numerous methods have been proposed and protocols developed in attempts to reduce healthcare costs. Statistical-based systems, including the Revised Cardiac Risk Index and American College of Surgeons National Surgical Quality Improvement Program (ACS-NSQIP) Surgical Risk Calculator, have been used for predicting risk [11-13]. However, the effect of preoperative use of opioids, sedatives, and antidepressants has not been examined in colorectal surgery. We hypothesize the use of these medications is an important predictor in increased surgical admission costs. The objective of this study is to evaluate the impact of preoperative opioid, sedative, and antidepressant use on hospital costs in patients undergoing colorectal resection at a single institution.

\section{Methods}

\section{Study population}

This study was a single-institution retrospective record review. The study received approval from the University of Kentucky, Office of Research Integrity Institutional Review Board, with exemption from patient consent and met the guidelines of the responsible government agency. The local University of Kentucky ACS-NSQIP database was queried for adult patients (age $\geq 18$ years) undergoing open or laparoscopic, partial or total, colectomy or proctectomy at the University of Kentucky from January 1, 2013 to December 31, 2016. Patients admitted after trauma, who were under 18 years of age, and who had a major surgical procedure within 30 days prior to the index surgery were excluded. The NSQIP database has been used extensively in literature, is peer regulated by surgeons, and allows for a common interpretation of risk factors and 30-day outcome measurements across studies [14].

Data obtained from the NSQIP database included patient characteristics (gender, age, race, American Society of Anesthesiology [ASA] classification, body mass index [BMI], functional status, smoking status, and medical comorbidities including congestive heart failure [CHF], chronic obstructive pulmonary disease [COPD], diabetes, renal failure, ascites, and hypertension), preoperative laboratory values (white blood cells, sodium, BUN, creatinine, albumin, hematocrit, platelets, INR, alkaline phosphatase, and bilirubin levels), and surgical variables (emergency status, operative indication, procedure type, wound classification, ostomy creation, and operative duration).

Individual patient medical records were reviewed to determine the preoperative use of specific opioids, sedatives, and antidepressants based on the medication reconciliation (which was finalized and confirmed by direct communication with the patient's pharmacy as well as a pharmacist to patient interview). Preoperative use was defined as a medication listed on the Medication Reconciliation Document on admission by the patient and discussed with a member of pharmacy staff. Hospital cost data from the index admission were captured by the hospital cost accounting system (Allscripts EPSi Version 7.5 FP2, Chicago, IL; Allscripts Healthcare, LLC, Chesterfield, MO) and matched to the cases identified by the NSQIP query. Total hospital costs included operating room services and supplies to include holding room, operating room, post-anesthesia care, as well as ward accommodations, intensive care, pharmacy, diagnostics, ancillary treatments such as physical therapy, and blood. Total hospital costs included both direct (supplies, nursing, and equipment) and indirect costs (administration, facility, and other overhead), but did not include professional fees.

Outcome measures included costs related to normal accommodations, supplies, operating room, pharmacy, diagnostics, intensive care, and ancillary therapies. These costs were aggregated to give us Total Hospital Cost. All patient characteristics, surgical characteristics, and outcomes recorded were strictly defined by the ACS-NSQIP 2017 Participant Use Data File [15]. There were no patients who had missing outcomes data or were lost to follow-up as only complete 30-day follow-up data was recorded into our local NSQIP database.

\section{Statistical analysis}

All ACS-NSQIP categorical patient characteristic, operative risk, and outcome variables were compared in medication groups using chi-square tests or Fisher's exact tests, and continuous variables were compared using Mann-Whitney U tests. A $p$ value of $\leq 0.05$ was considered the threshold for statistical significance in the bivariate calculations.

ACS-NSQIP patient and operative risk factors were considered in a backward multivariable logistic regression of total costs ( $p$ for entry $\leq 0.05$, for exit $\geq 0.10$ ). Medication use variables were then added into the model in the presence of the independent risk factors.

\section{Results}

A total of 1185 colorectal procedures were performed at a single site by 30 different surgeons with volumes ranging from 1 to 497 cases. The mean age was $55.8 \pm 15.5$ years and median age was 57. Six-hundred two (50.8\%) were female, $134(11.3 \%)$ minority race, and $59(0.5 \%)$ were of known 
Hispanic ethnicity. Seven-hundred ninety-three (66.9\%) were elective procedures, and $22.5 \%$ were emergent.

\section{Patient preoperative characteristics predictive of hospital cost}

The mean total hospital cost (MTHC) for the cohort, measured in $\$ 1000 \mathrm{~s}$, was $25.7 \pm 30.5$. MTHC was 19.8 for elective cases which increased for non-elective, non-emergent (32.0), and emergent (40.4). Transfer status was also significantly associated with MTHC as patients admitted from home had lower cost than those from outside hospitals, emergency departments, and nursing homes. MTHC increased with increasing ASA class. Functional status, pulmonary status, preoperative inflammatory response, and other significant medical comorbidities were associated with increased MTHC as listed in Table 1.

Patient characteristics that did not vary with MTHC included sex, race or ethnicity, current smoking status, ascites, chronic use of steroids, recent weight loss greater than $10 \%$, and BMI.

Preoperative laboratory values associated with MTHC were assessed. Uremia, hyponatremia and hypernatremia, elevated creatinine, hypoalbuminemia, leukocytosis and leukopenia, anemia, and thrombocytopenia were associated with increased MTHC. Thrombocytosis was associated with decreased MTHC. The mean total hospital costs associated with abnormal laboratory values are listed in Table 2.

After backwards multiple regression, independent patient predictors of cost in order of predictive strength were ASA class, preoperative inflammatory response, functional status, existing open wound, hypoalbuminemia, disseminated cancer, leukocytosis, leukopenia, bleeding disorders, and dyspnea.

\section{Operative characteristics predictive of hospital cost}

Open colectomy procedures (CPT code beginning with 4414- and 4415-) had increased MTHC, while laparoscopic colectomy procedures (CPT code beginning with 4420and 4421-) had decreased MTHC. A full listing of operative procedures and costs are listed in Table 3. Increasing operative duration was also predictive of increased MTHC. Clean/contaminated wounds had lower MTHC (20.9) than contaminated (26.2) and dirty/infected wounds (30.0) as did wound closure techniques. Fully closed wounds had MTHC of 23.4, while MTHC increased with closure of only deep layers (42.5) and no closure (75.9). Preoperative transfusion within $72 \mathrm{~h}$ was predictive of increased MTHC.

With independent preoperative risk factors fixed in the model, independent operative predictors of cost were procedure group (first four digits of CPT code), duration of operation, wound closure technique, and transfusion requirement.

\section{Hospital costs by drug class}

Three-hundred twenty-seven (27.6\%) patients took opioids (OPD), 219 (18.5\%) took sedatives (SDT), and 329 (27.8\%) took antidepressants (ADM) preoperatively. All classes of medications studied were associated with increased MTHC (30.8 vs 23.6 for OPD, 31.6 vs 24.4 for SDT, and 30.7 vs 23.8 for ADM).

Patients taking OPD were found to have increased costs compared to non-users for all sub-costs in the analysis. Mean accommodation costs and supply costs were each $\$ 970$ more than non-users. ICU costs were $\$ 2,130$ more for OPD users. OPD users had a mean increase of $\$ 1,310$ in pharmacy costs compared to non-users. A complete listing of cost differences per category can be seen in Table 4 . When adjusted for independent preoperative and operative risks for increased cost on multivariate analysis, patients taking OPD had a cost increase of $\$ 1,173$ (regression coefficient $1.07,95 \%$ confidence interval (CI) $1.01-1.14, p=0.030$ ).

Similar to OPD users, patients taking SDT were associated with increased mean cost of various subcategories. SDT users saw a mean increase of $\$ 2,740$ in ICU costs and $\$ 1,380$ in pharmacy costs. Complete listing of cost differences per category can be seen in Table 4 . When controlled for significant variables on multivariate analysis, patients taking SDT had a mean cost increase of $\$ 1,843$ (regression coefficient $1.11,95 \%$ CI 1.03-1.19, $p=0.004)$.

Antidepressant use followed a comparable trend as OPD and SDT use. Mean bed cost was $\$ 1,010$ higher than non-users. ICU costs were $\$ 2,030$ higher on average and pharmacy costs were increased by $\$ 1,630$. Complete listing of cost differences by category can be found in Table 4 . On multivariate analysis, ADM users had a mean increase of $\$ 838$ (regression coefficient $1.05,95 \%$ CI $0.99-1.12$, $p=0.102$ ).

An interaction term between the drug use classes in the multivariable regression was not a significant predictor of MTHC.

\section{Discussion}

Preoperative use of OPD, SDT, and ADM has been increasing. Nearly a third of our patients undergoing colorectal surgery used OPD and ADM preoperatively, and over one in five took SDT. Optimizing patients preoperatively is a key surgical principle and has classically focused on modifiable risk factors, such as tobacco use, obesity, and functional status, as well as cardiopulmonary status and nutrition. Only recently has preoperative usage of specific medications been studied as it relates to postoperative outcomes $[16,17]$. OPD and SDT have been identified as risk factors for poor surgical outcomes [18, 
Table 1 Patient preoperative characteristics predictive of hospital costs

\begin{tabular}{|c|c|c|c|}
\hline Variable & $n$ & $\begin{array}{l}\text { Mean total hospital costs } \\
\$ 000 \text { 's (SD) }\end{array}$ & $p$ value \\
\hline All patients & 1185 & $25.7(30.5)$ & \\
\hline Presentation & & & $<.001$ \\
\hline Same-day elective & 793 & 19.8 & \\
\hline Not elective \& not emergent & 125 & 32.0 & \\
\hline Emergency & 267 & 40.4 & \\
\hline Transfer status & & & $<.001$ \\
\hline Not transferred (admitted from home) & 912 & 21.6 & \\
\hline Outside emergency department & 164 & 34.6 & \\
\hline From acute care hospital inpatient & 80 & 46.3 & \\
\hline Nursing home-chronic care-intermediate care & 15 & 50.2 & \\
\hline Other/Unknown & 14 & 48.4 & \\
\hline Age quartile, year & & & $<.001$ \\
\hline$\leq 47$ & 317 & 30.2 & \\
\hline $48-57$ & 292 & 35.7 & \\
\hline $58-67$ & 307 & 28.7 & \\
\hline $68+$ & 269 & 25.9 & \\
\hline ASA physical status classification & & & $<.001$ \\
\hline $\mathrm{I}-\mathrm{II}$ & 373 & 17.5 & \\
\hline III & 695 & 24.8 & \\
\hline IV-V & 117 & 57.4 & \\
\hline Diabetes & & & $<.001$ \\
\hline Non-insulin & 133 & 27.2 & \\
\hline Insulin & 90 & 34.3 & \\
\hline Preoperative functional status & & & $<.001$ \\
\hline Partially dependent & 65 & 47.6 & \\
\hline Totally dependent & 17 & 70.3 & \\
\hline Preoperative inflammatory response & & & $<.001$ \\
\hline None & 1002 & 20.5 & \\
\hline SIRS & 26 & 29.7 & \\
\hline Sepsis & 98 & 42.3 & \\
\hline Septic shock & 59 & 74.4 & \\
\hline Dyspnea & & & $<.001$ \\
\hline No & 984 & 26.2 & \\
\hline Moderate exertion & 175 & 26.6 & \\
\hline At rest & 26 & 99.7 & \\
\hline Severe COPD & 119 & 35.9 & $<.001$ \\
\hline Preoperative ventilator & 25 & 89.3 & $<.001$ \\
\hline $\mathrm{CHF}$ & 28 & 58.3 & $<.001$ \\
\hline Medically treated HTN & 628 & 29.8 & $<.001$ \\
\hline Renal failure & 7 & 114.8 & $<.001$ \\
\hline Dialysis & 19 & 54.9 & $<.001$ \\
\hline Disseminated cancer & 162 & 27.7 & .009 \\
\hline Preoperative open wound & 63 & 41.0 & $<.001$ \\
\hline Bleeding disorder & 109 & 45.8 & $<.001$ \\
\hline Preoperative transfusion & 18 & 65.8 & $<.001$ \\
\hline
\end{tabular}

SIRS systemic inflammatory response syndrome, $C O P D$ chronic obstructive pulmonary disease, $C H F$ congestive heart failure, $H T N$ hypertension 
Table 2 Patient preoperative lab values predictive of hospital costs

\begin{tabular}{llll}
\hline Variable & $n$ & $\begin{array}{l}\text { Mean total hospital } \\
\text { costs } \$ 000 \text { 's (SD) }\end{array}$ & $p$ value \\
\hline All patients & 1185 & $25.7(30.5)$ & \\
BUN $>40 \mathrm{mg} / \mathrm{dL}$ & 53 & 62.2 & $<.001$ \\
Sodium $<135 \mathrm{mmol} / \mathrm{L}$ & 70 & 35.3 & $<.001$ \\
Sodium $>145 \mathrm{mmol} / \mathrm{L}$ & 18 & 37.3 & $<.001$ \\
Creatinine $>1.2 \mathrm{mg} / \mathrm{dL}$ & 160 & 43.1 & $<.001$ \\
Albumin $<3.5 \mathrm{~g} / \mathrm{dL}$ & 461 & 35.1 & $<.001$ \\
WBC $\leq 4500 / \mathrm{mm}{ }^{3}$ & 78 & 35.1 & $<.001$ \\
WBC $>11,000 / \mathrm{mm}^{3}$ & 241 & 38.5 & $<.001$ \\
HCT $<38 \%$ & 519 & 30.9 & $<.001$ \\
Platelets $<150,000 / \mathrm{mm}^{3}$ & 94 & 45.7 & $<.001$ \\
Platelets $>400,000 / \mathrm{mm}^{3}$ & 92 & 21.3 & $<.001$ \\
\hline
\end{tabular}

$B U N$ blood, urea, nitrogen, $W B C$ white blood cell, $H C T$ hematocrit

19]. When adjusted for all significant independent preoperative and perioperative risk factors, OPD and SDT significantly increased MTHC. ADM use also showed a trend toward increased MTHC though it did not reach statistical significance. These findings identify preoperative use of OPD and SDT as independent risk factors for increased hospital costs relating to colorectal surgery.
The OPD epidemic has seen a significant increase in prescription and illicit OPD use in Kentucky and surrounding states to levels higher than national averages [20,21]. OPD has been associated with increased postoperative complications and length of stay in orthopedic and abdominal surgery literature [10, 22, 23]. Patients using OPD have been shown to be at higher risk for pneumonia and respiratory failure. This is due to respiratory depression and is worsened in patients with COPD. OPD has also been associated with factors prolonging length of stay, including postoperative nausea and vomiting, prolonged ileus, and urinary retention. These have also led to increased morbidity and mortality $[10,24,25]$. Our results demonstrate increased cost in effectively all aspects of hospitalization, including costs relating to operating room, supplies, intensive care, pharmacy, diagnostics, and ancillary treatments. After adjusting for known risk factors, preoperative OPD use increased MTHC by nearly $\$ 1,200$.

Costs related to operating room, intensive care, pharmacy, diagnostics, and ancillary treatments were increased in patients taking SDT preoperatively. As previously stated, SDT has been found to be an independent risk factor for adverse outcomes in non-cardiac surgery [18]. Patients taking these medications also have been found to have increased operative times, increased length of stay, and
Table 3 Operative characteristics predictive of hospital costs

\begin{tabular}{|c|c|c|c|}
\hline Variable & $n$ & $\begin{array}{l}\text { Mean total hospital } \\
\text { costs } \$ 000 \text { 's (SD) }\end{array}$ & $p$ value \\
\hline All patients & 1185 & $25.7(30.5)$ & \\
\hline Procedure group & & & $<.001$ \\
\hline Open partial colectomy & 405 & 33.6 & \\
\hline Total abdominal colectomy & 58 & 50.3 & \\
\hline Proctectomy & 47 & 22.9 & \\
\hline Lap partial colectomy & 425 & 17.7 & \\
\hline Lap total abdominal colectomy & 75 & 18.3 & \\
\hline Lap proctectomy & 76 & 20.6 & \\
\hline Ostomy & 650 & 29.3 & $<.001$ \\
\hline Duration of operation, incision to close, mins. & & & $<.001$ \\
\hline$\leq 120$ & 322 & 22.5 & \\
\hline $121-180$ & 409 & 25.3 & \\
\hline $181-240$ & 248 & 27.2 & \\
\hline $241+$ & 206 & 30.1 & \\
\hline Wound class & & & $<.001$ \\
\hline Clean/Contaminated & 706 & 20.9 & \\
\hline Contaminated & 276 & 26.2 & \\
\hline Dirty/Infected & 203 & 41.9 & \\
\hline Wound closure & & & $<.001$ \\
\hline All layers of incision (deep and superficial) fully closed & 1111 & 23.4 & \\
\hline Only deep layers closed; superficial left open & 33 & 42.5 & \\
\hline No layers of incision are surgically closed & 41 & 75.9 & \\
\hline Transfused w/in $72 \mathrm{~h}$ of incision & 146 & 56.3 & $<.001$ \\
\hline
\end{tabular}


Table 4 Costs in drug use and non-use cohorts; by cost center (\$000's)

\begin{tabular}{|c|c|c|c|c|c|c|c|c|c|c|}
\hline \multirow[t]{2}{*}{ Cost center } & \multirow[t]{2}{*}{ All pts. } & \multicolumn{2}{|c|}{ Opioid } & \multirow{2}{*}{$\begin{array}{l}\text { Diff. } \\
p^{-}\end{array}$} & \multicolumn{2}{|c|}{ Sedative } & \multirow{2}{*}{$\begin{array}{l}\text { Diff. } \\
p-\end{array}$} & \multicolumn{2}{|c|}{$\begin{array}{l}\text { Antidepres- } \\
\text { sant }\end{array}$} & \multirow{2}{*}{$\begin{array}{l}\text { Diff. } \\
p-\end{array}$} \\
\hline & & No & Yes & & No & Yes & & No & Yes & \\
\hline No. pts. & 1185 & 828 & 327 & value & 966 & 219 & value & 856 & 329 & value \\
\hline Mean total costs (SD) & $25.7(30.5)$ & 23.6 & 30.8 & $<.001$ & 24.4 & 31.6 & .002 & 23.8 & 30.7 & .001 \\
\hline Bed costs & $6.43(6.01)$ & 6.14 & 7.11 & .011 & 6.28 & 7.11 & .065 & 6.15 & 7.16 & .010 \\
\hline Med-Surg supply costs & $5.20(5.39)$ & 4.91 & 5.88 & .005 & 5.10 & 5.66 & .165 & 5.05 & 5.61 & .109 \\
\hline OR costs & $4.82(2.63)$ & 4.70 & 5.09 & .022 & 4.74 & 5.18 & .023 & 4.79 & 4.91 & .486 \\
\hline ICU costs & $3.59(10.4)$ & 2.95 & 5.08 & .001 & 3.08 & 5.82 & $<.001$ & 3.03 & 5.06 & .003 \\
\hline Pharmacy costs & $2.66(6.43)$ & 2.27 & 3.58 & .001 & 2.41 & 3.79 & .004 & 2.21 & 3.84 & $<.001$ \\
\hline Diagnostics costs & $2.06(3.82)$ & 1.82 & 2.62 & .001 & 1.95 & 2.57 & .028 & 1.79 & 2.78 & $<.001$ \\
\hline Ancill. Trx. costs & $0.74(2.30)$ & 0.59 & 1.08 & .001 & 0.64 & 1.17 & .002 & 0.62 & 1.06 & .003 \\
\hline Blood costs & $0.23(0.97)$ & 0.20 & 0.32 & .043 & 0.23 & 0.27 & .546 & 0.21 & 0.29 & .235 \\
\hline
\end{tabular}

increased readmissions [26]. This could be attributed to the effects of SDT on respiratory and cognitive functions which have been demonstrated to contribute to poor surgical outcomes [27-29]. Chronic use of SDT has also been shown to increase postoperative delirium, and decrease adequate pain control and overall prolonged surgical recovery [30-33]. Adverse events, such as surgical site infection, lead to a need for further procedures, longer hospital stays, and amplified care requirements. After adjusting for significant independent risk factors, SDT use increased MTHC by over $\$ 1,800$.

ADM use has been shown to be associated with increased adverse events, prolonged LOS, and higher morbidity and mortality $[34,35]$. Our study showed significantly increased costs relating to bed costs, intensive care, pharmacy, diagnostics, and ancillary therapies. Nonetheless, our multivariate analysis did not demonstrate that preoperative ADM use independently increases MTHC. Also, any combined use of multiple medication classes did not have a synergistic effect on MTHC greater than the risk of each medication class alone.

Prior to this study, the association between preoperative medication use and postoperative hospital costs had not been studied in colorectal surgery literature. It has been reported that preoperative OPD use has increased overall healthcare costs [7]. Reports from spine surgery literature have shown increased costs following cervical and lumbar fusions [36, 37]. Elective abdominal general surgery procedures have also shown increased hospital costs as well as overall postoperative costs [10]. However, no study to date has investigated the relationship between preoperative OPD and SDT use to all-comers in colorectal surgery resections.

Given our results, the preoperative use of OPD and SDT should be considered in the preoperative risk stratification and optimization of colorectal surgery patients. Enhanced Recovery after Surgery (ERAS) is a surgical optimization program that systematically works to decrease surgical complications, LOS, and costs [38-40]. While ERAS has previously primarily focused on perioperative OPD use, our findings indicate that SDT use may also be considered in the prediction of increased perioperative costs. Provider's knowledge of preoperative use of these medications may allow for expectant management of postoperative complications and reduce costs.

The ACS-NSQIP database allows for comparison with other studies performed through its database, given the precise definition of each outcome variable and homogeneity of the data series within a fixed timeframe. However, there are limitations to this study. We cannot prove causality within a retrospective study despite demonstrating a strong association between SDT and increased MTHC. Also, with the study performed at a single institution, the results may not apply to all centers. There lies the possibility of unmeasured confounders as well, including high-risk comorbid conditions or clinical risk factors among medication users which could influence morbidity and mortality more than the medication itself. Multivariate analysis will adjust for known confounders, but it is impossible to capture all risk factors associated with outcomes and cost. In the same light, preoperative medication users could be higher risk than captured in our bivariate analysis. Under these circumstances, recognition of preoperative medication use can aide in identifying patients with higher risk of increased cost. Two sources of potential bias were identified. First, not all preoperative and operative risk factors were captured in our study. Examples could be patient compliance and socioeconomic status. However, the NSQIP database is one of the most robust clinical datasets available, including blood tests and other granular clinical variables. Additionally, there is unknown treatment bias related to this retrospective review that could alter the outcomes resulting from unknown surgical decision making as it relates to technique, approach, and indications. Our study did include multiple surgeons from different services which partially mitigates selection bias. 
Further limitations include the lack of both specific dosage and length of use for each medication preoperatively in our data analysis. This would allow us to stratify users based on these factors and improve generalizability. Unfortunately, we were limited by the capabilities of the medication record, which is currently being improved to allow linkage to a statewide electronic reporting system. Lastly, preoperative illicit use of these medications was not captured in our database. With the current state of the OPD epidemic and illicit use of SDT, we may be significantly underestimating the true amount of preoperative SDT use as well as OPD use. These could skew the data analysis and give falsely low results. Certainly, future randomized prospective studies are warranted to further this project and also to identify the impact of medication dose or chronicity on healthcare costs.

\section{Conclusion}

OPD, SDT, and ADM use is increasingly prevalent and can be used to predict increased total hospital costs in patients undergoing colorectal resections. These increased costs, in combination with previous findings of risk for adverse outcomes, underscore the importance of preoperative evaluation of drug usage for both risk prediction and optimization.

Funding This research did not receive any specific grant from funding agencies in the public, commercial, or not-for-profit sectors.

\section{Compliance with ethical standards}

Disclosures The authors Nicholas Jackson, Tong Gan, Daniel L. Davenport, Doug R. Oyler, Laura M. Ebbitt, B. Mark Evers, and Avinash $S$. Bhakta have no conflicts of interest to disclose or financial ties to disclose.

\section{References}

1. Maust DT, Lin LA, Blow FC (2019) Benzodiazepine use and misuse among adults in the United States. Psychiatr Serv 70:97-106

2. Pratt LB, Brody DJ, Gu Q (2017) Antidepressant use among persons aged 12 and over: United States, 2011-2014. NCHS Data Brief 283:1-8

3. Kenan K, Mack K, Paulozzi L (2012) Trends in prescriptions for oxycodone and other commonly used opioids in the United States, 2000-2010. Open Med 6:e41-47

4. Paulozzi LJ, Mack KA, Hockenberry JM (2014) Variation among states in prescribing of opioid pain relievers and benzodiazepinesUnited States, 2012. J Saf Res 51:125-129

5. Kolodny A, Courtwright DT, Hwang CS, Kreiner P, Eadie JL, Clark TW, Alexander GC (2015) The prescription opioid and heroin crisis: a public health approach to an epidemic of addiction. Annu Rev Public Health 36:559-574
6. Vietri J, Masters ET, Barsdorf AI, Mardekian J (2018) Cost of opioid medication abuse with and without tampering in the USA. Clinicoecon Outcomes Res 10:433-442

7. Chang HY, Kharrazi H, Bodycombe D, Weiner JP, Alexander GC (2018) Healthcare costs and utilization associated with high-risk prescription opioid use: a retrospective cohort study. BMC Med 16:69

8. Florence CS, Zhou C, Luo F, Xu L (2016) The economic burden of prescription opioid overdose, abuse, and dependence in the United States, 2013. Med Care 54:901-906

9. Hilliard PE, Waljee J, Moser S, Metz L, Mathis M, Goesling J, Cron D, Clauw DJ, Englesbe M, Abecases G, Brummett CM (2018) Prevalence of preoperative opioid use and characteristics associated with opioid use among patients presenting for Surgery. JAMA Surg 153:929-937

10. Waljee JF, Cron DC, Steiger RM, Zhong L, Englesbe MJ, Brummett CM (2017) Effect of preoperative opioid exposure on healthcare utilization and expenditures following elective abdominal surgery. Ann Surg 265:715-721

11. Liu Y, Cohen ME, Hall BL, Ko CY, Bilimoria KY (2016) Evaluation and enhancement of calibration in the American College of Surgeons NSQIP surgical risk calculator. J Am Coll Surg 223:231-239

12. Lee TH, Marcantonio ER, Mangione CM, Thomas EJ, Polanczyk CA, Cook EF, Sugarbaker DJ, Donaldson MC, Poss R, Ho KK, Ludwig LE, Pedan A, Goldman L (1999) Derivation and prospective validation of a simple index for prediction of cardiac risk of major noncardiac surgery. Circulation 100:1043-1049

13. Bilimoria KY, Liu Y, Paruch JL, Zhou L, Kmiecik TE, Ko CY, Cohen ME (2013) Development and evaluation of the universal ACS NSQIP surgical risk calculator: a decision aid and informed consent tool for patients and surgeons. J Am Coll Surg 217:833-842

14. Maggard-Gibbons M (2014) The use of report cards and outcome measurements to improve the safety of surgical care: the American College of Surgeons National Surgical Quality Improvement Program. BMJ Qual Saf 23:589-599

15. User Guide for the 2017 ACS NSQIP Participant Use Data File (PUF). 2018. https://www.facs.org/ /media/files/quality\%2520p rograms/nsqip/nsqip_puf_userguide_2017.ashx. Accessed June 22,2020

16. Garcia-Miguel FJ, Serrano-Aguilar PG, Lopez-Bastida J (2003) Preoperative assessment. Lancet 362:1749-1757

17. Gan T, Jackson NA, Castle JT, Davenport DL, Oyler DR, Ebbitt LM, Evers BM, Bhakta AS (2020) A retrospective review: patient-reported preoperative prescription opioid, sedative, or antidepressant use is associated with worse outcomes in colorectal surgery. Dis Colon Rectum 63:965-973

18. Ward N, Roth JS, Lester CC, Mutiso L, Lommel KM, Davenport DL (2015) Anxiolytic medication is an independent risk factor for 30-day morbidity or mortality after surgery. Surgery 158:420-427

19. Cron DC, Englesbe MJ, Bolton CJ, Joseph MT, Carrier KL, Moser SE, Waljee JF, Hilliard PE, Kheterpal S, Brummett CM (2017) Preoperative opioid use is independently associated with increased costs and worse outcomes after major abdominal surgery. Ann Surg 265:695-701

20. Nelson LS, Juurlink DN, Perrone J (2015) Addressing the opioid epidemic. JAMA 314:1453-1454

21. Manchikanti L, Helm S 2nd, Fellows B, Janata JW, Pampati V, Grider JS, Boswell MV (2012) Opioid epidemic in the United States. Pain Phys 15(3 Suppl):ES9-ES38

22. Li Y, Stocchi L, Cherla D, Liu X, Remzi FH (2016) Association of preoperative narcotic use with postoperative complications and prolonged length of hospital stay in patients with Crohn disease. JAMA Surg 151:726-734 
23. Cozowicz C, Olson A, Poeran J, Morwald EE, Zubizarreta N, Girardi FP, Hughes AP, Mazumdar M, Memtsoudis SG (2017) Opioid prescription levels and postoperative outcomes in orthopedic surgery. Pain 158:2422-2430

24. Sayal P, Bateman BT, Menendez M, Eikermann M, Ladha KS (2018) Opioid use disorders and the risk of postoperative pulmonary complications. Anesth Analg 127:767-774

25. Chung W-S, Lai C-Y, Lin C-L, Kao C-H (2015) Adverse respiratory events associated with hypnotics use in patients of chronic obstructive pulmonary disease: a population-based case-control study. Medicine 94:e1110-e1110

26. Neff C, Totten C, Plymale M, Oyler DR, Davenport D, Roth JS (2018) Associations between anxiolytic medications and ventral hernia repair. Hernia 22:753-757

27. Raats JW, van Eijsden WA, Crolla RMPH, Steyerberg EW, van der Laan L (2015) Risk factors and outcomes for postoperative delirium after major surgery in elderly patients. PLoS ONE 10:e0136071-e0136071

28. American Geriatrics Society Expert Panel on Postoperative Delirium in Older Adults (2015) Postoperative delirium in older adults: best practice statement from the American Geriatrics Society. J Am Coll Surg 220:136-148.e1

29. Brown 4th C, Deiner S (2016) Perioperative cognitive protection. Br J Anaesth 117(suppl_3):iii52-iii61

30. Sutherland AM, Katznelson R, Clarke HA, Tait G, Beattie WS (2014) Use of preoperative antidepressants is not associated with postoperative hospital length of stay. Can J Anaesth 61:27-31

31. Orri M, Boleslawski E, Regimbeau J-M, Barry C, Hassler C, Gregoire E, Bachellier P, Scatton O, Mabrut JY, Adham M, Vibert E, Revah-Levy A, Farges O (2015) Influence of depression on recovery after major noncardiac surgery. Ann Surg 62:882-899; discussion 889-890

32. Kudoh A, Takase H, Takahira Y, Takazawa T (2004) Postoperative confusion increases in elderly long-term benzodiazepine users. Anesth Analg 99:1674-1678

33. Brown CH, LaFlam A, Max L, Wyrobek J, Neufeld KJ, Kebaish KM, Cohen DB, Walston JD, Hogue CW, Riley LH (2016)
Delirium after spine surgery in older adults: incidence, risk factors, and outcomes. J Am Geriatr Soc 64:2101-2108

34. Vashishta R, Kendale SM (2019) Relationship between preoperative antidepressant and antianxiety medications and postoperative hospital length of stay. Anesth Analg 128:248-255

35. Auerbach AD, Vittinghoff E, Maselli J, Pekow PS, Young JQ, Lindenauer PK (2013) Perioperative use of selective serotonin reuptake inhibitors and risks for adverse outcomes of surgery. JAMA Intern Med 173:1075-1081

36. Jain N, Brock JL, Phillips FM, Weaver T, Khan SN (2018) Chronic preoperative opioid use is a risk factor for increased complications, resource use, and costs after cervical fusion. Spine $\mathbf{J}$ 18:1989-1998

37. Jain N, Phillips FM, Weaver T, Khan SN (2018) Preoperative chronic opioid therapy: a risk factor for complications, readmission, continued opioid use and increased cost after one- and twolevel posterior lumbar fusion. Spine 43:1331-1338

38. Greco M, Capretti G, Beretta L, Gemma M, Pecorelli N, Braga M (2014) Enhanced recovery program in colorectal surgery: a meta-analysis of randomized controlled trials. World J Surg 38:1531-1541

39. Muller S, Zalunardo MP, Hubner M, Clavien PA, Demartines N, Zurich Fast Track Study Group (2009) A fast-track program reduces complications and length of hospital stay after open colonic surgery. Gastroenterology 136:842-847

40. Roulin D, Donadini A, Gander S, Griesser A-C, Blanc C, Hubner M, Schafer M, Demartines N (2013) Cost-effectiveness of the implementation of an enhanced recovery protocol for colorectal surgery. Br J Surg 100:1108-1114

Publisher's Note Springer Nature remains neutral with regard to jurisdictional claims in published maps and institutional affiliations. 